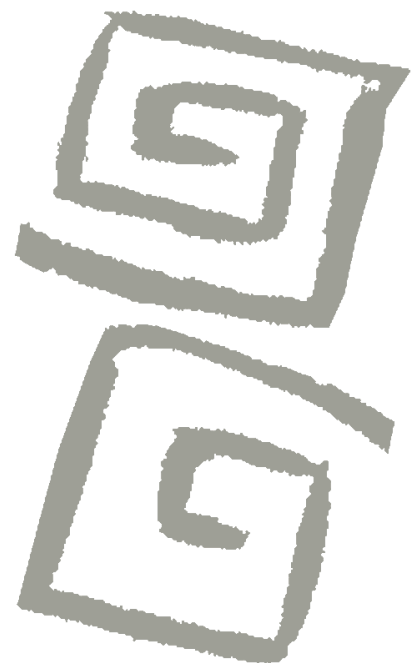

\title{
Tensiones y contradicciones en la intervención gubernamental para la promoción de la lactancia materna
}

\author{
Tensions and contradictions in government \\ interventions for the promotion of breastfeeding \\ Rosa María Ramos Rodríguez', María Gabriela Hernández González²
}

'Doctora en Antropología. Investigadora titular, Instituto de Investigaciones Antropológicas, Universidad Nacional Autónoma de México, México. $\square$ iD

${ }^{2}$ Maestra en Antropología. Doctoranda en Antropología, Universidad Nacional Autónoma de México, México. $\triangle$ iD
RESUMEN Con el propósito de esclarecer el abandono de la lactancia materna en zonas rurales de México, se aborda el modelo biomédico vigente y las políticas y acciones para la promoción de la lactancia materna que se derivan de su enfoque teórico, y se discuten las estrategias operativas del programa gubernamental de beneficio social Oportunidades. Para ello se retoman los testimonios de 39 madres jóvenes lactantes, 11 mujeres madres abuelas y 12 miembros del personal sanitario de la población náhuatl de Cuentepec, Morelos, México, recabados en un trabajo de investigación previo, realizado entre 2008 y 2009. Se constató que el modelo biomédico, que permea todas las acciones de promoción de lactancia materna, cosifica a las personas, limita la comunicación, devalúa los saberes tradicionales de las mujeres e impone un discurso que paulatinamente desalienta dicha práctica. Se propone adoptar un cambio epistémico del pensamiento biomédico que transite del paradigma de la simplicidad al de la complejidad, para lograr un acercamiento mayor a la comprensión de los procesos bio-psico-socio-culturales propios del ser humano.

PALABRAS CLAVES Lactancia Materna; Políticas Públicas; Planes y Programas de Salud; Población Indígena; México.

\begin{abstract}
With the purpose of shedding light on the decrease in the practice of breastfeeding in rural areas of Mexico, this article looks at the current biomedical model and the policies and actions to promote breastfeeding derived from the model's theoretical approach. The article also discusses operational strategies of the governmental social welfare program Oportunidades. For this purpose, the study utilizes the testimonies of 39 young breastfeeding mothers, 11 mothers and grandmothers and 12 members of the health staff in the Nahuatl population of Cuentepec, Morelos, Mexico, which were collected during a previous study in 2008 and 2009. It was found that the biomedical model, which permeates all actions to promote breastfeeding, reifies people, limits communication, devaluates women's traditional knowledge and imposes a discourse that gradually discourages the practice of breastfeeding. The article's proposal is to adopt an epistemic change in biomedical thought that shifts from a paradigm of simplicity to one of complexity, with the purpose of achieving a greater understanding of the biopsycho-socio-cultural processes of human beings.
\end{abstract}

KEY WORDS Breast Feeding; Public Policies; Health Programs and Plans; Indigenous Population; Mexico. 


\section{INTRODUCCIÓN}

Debido al rápido crecimiento físico y a la acelerada multiplicación de las interconexiones nerviosas presentes en el desarrollo del cerebro humano ${ }^{(1,2,3)}$, los niños son extremadamente lábiles durante el primer año de vida, lo que propicia su elevada morbilidad y mortalidad. Por ello, para garantizar su supervivencia y desarrollo, es fundamental, entre otros aspectos, que las prácticas de alimentación infantil sean adecuadas para sus requerimientos durante, al menos, los dos primeros años de la vida ${ }^{(4)}$.

Existen múltiples estudios dedicados al tema de la lactancia materna, en los que se sustenta la idoneidad de amamantar o de alimentar con leche humana, priorizando sus aspectos biológicos ${ }^{(5,6)}$. La gran mayoría de ellos abordan las particularidades de la composición bioquímica de dicho fluido y su impacto benéfico para la nutrición, el sistema inmunológico de los infantes ${ }^{(7)}$ y su crecimiento y desarrollo físico y mental ${ }^{(4,8,9)}$. A su vez, otros han demostrado ampliamente que el abandono de la lactancia materna impacta negativamente tanto en la esfera física, como psíquica del niño y de la madre, así como en la economía familiar y en los costos de la atención sanitaria derivados de las prácticas inadecuadas en la lactancia ${ }^{(10)}$.

Aunque la lactancia materna es universalmente concebida como beneficiosa ${ }^{(11)}$, paradójicamente, en el mundo y en particular en México, su práctica está registrando una marcada tendencia descendente. De hecho, los resultados de las últimas dos encuestas sobre salud y nutrición realizadas en México indican un alarmante deterioro en la prevalencia de cualquier tipo de lactancia, especialmente, de la lactancia materna exclusiva. Ello posiciona al país en el último lugar en Latinoamérica en el ejercicio de dicha práctica, pues solo el $14,4 \%$ de las mujeres amamantan exclusivamente con su leche hasta los seis meses. Aún más, en los últimos doce años, esta tendencia se ha observado en las comunidades rurales, las cuales gozaban de las tasas más altas de lactancia materna, la cual ha descendido del $36,9 \%$ al $18,5 \%{ }^{(12)}$. En México, el abatimiento de esta práctica es un proceso que viene ocurriendo desde hace años y aunque se ha tratado de explicar estimando asociaciones estadísticas derivadas de la información de las encuestas, los resultados no contribuyen a su esclarecimiento ${ }^{(13)}$.

$\mathrm{Si}$ bien estas investigaciones son pertinentes y propicias, adolecen de una visión que reconozca a la lactancia materna como un proceso bio-psico-socio-cultural ${ }^{(14,15,16)}$ partícipe de la complejidad que caracteriza al Homo sapiens sapiens $s^{(3,17,18,19)}$. Por ello, para la completa comprensión de este proceso alimentario, se requiere que sea abordado bajo una mirada que ponga atención en las interacciones que se establecen entre los procesos biológicos, sociales y culturales ${ }^{(5,20,21,22)}$ así como en los contextos sociohistóricos en los que ocurre.

\section{ACERCA DE LA INVESTIGACIÓN}

Este trabajo tiene por objetivo deliberar en torno al modelo biomédico vigente y las políticas y acciones para la promoción de la lactancia materna que se derivan de su enfoque teórico, contribuyendo con ello al esclarecimiento del abandono de dicha práctica en zonas rurales. La argumentación desemboca en la discusión de las estrategias operativas del programa gubernamental de beneficio social Oportunidades, y se utilizan, a manera de ejemplo, los testimonios recabados en un trabajo de investigación previo llevado a cabo entre los meses de noviembre de 2008 a noviembre de 2009, en la población indígena náhuatl de Cuentepec, en el estado de Morelos, México ${ }^{(23)}$, caracterizada por tener un alto índice de marginación $^{(24)}$; estudio inscrito en el marco del Proyecto Enfoque antropológico de los Codeterminates del cambio en la condición nutricia en Cuentepec, Morelos, dentro de las actividades del Instituto de Investigaciones Antropológicas de la Universidad Nacional Autónoma de México. En dicho trabajo, empleando metodologías cualitativas, se 
observó, entre otros aspectos, la ejecución del programa Oportunidades y, en particular, las líneas de acción encaminadas a la promoción y apoyo de la lactancia materna. A través de entrevistas semiestructuradas, reuniones focales y observación participante se recopilaron los testimonios de 39 madres jóvenes lactantes, 11 mujeres madres abuelas y 12 miembros del personal sanitario que laboraban tanto en el Centro de Salud de Cuentepec como del Hospital Amigo del Niño y de la Madre localizado en Tetecala, Morelos. Para los fines del presente trabajo se seleccionaron algunos testimonios de las mujeres beneficiarias del mencionado programa y del personal de salud responsable de participar en su ejecución.

Previo a la realización de las entrevistas, a las personas que participaron en el estudio se les comunicaron los objetivos del proyecto de investigación y los procedimientos que se llevarían a cabo; después de haber recibido la información, aquellas que decidieron participar procedieron a firmar su consentimiento.

\section{SOBRE LAS ACCIONES GUBERNAMENTALES PARA LA PROMOCIÓN DE LA LACTANCIA MATERNA}

Aunque el concepto de política pública es polisémico, para lograr sincronía de conceptos, este trabajo se apega al propuesto por Velásquez que lo considera como “... un proceso integrador de decisiones, acciones, inacciones, acuerdos e instrumentos, adelantado por autoridades públicas con la participación eventual de los particulares, y encaminado a solucionar o prevenir una situación definida como problemática"(25), en este sentido puede incluir leyes, programas y proyectos; asignar recursos (gasto público e impuestos) o normar y legislar actividades ${ }^{(26)}$. Parsons ${ }^{(27)}$ señala que omitir acciones también forma parte de la política pública.

Cabe destacar que ciertos mecanismos para la construcción de las políticas sanitarias parten de la lógica neoliberal y los principios rectores planteados por el Fondo Monetario Internacional, por la Organización para la Cooperación y Desarrollo Económicos y por el Banco Mundial(28), es así que bajo tales principios los organismos internacionales responsables directamente de las políticas sanitarias como son la Organización Mundial de la Salud (OMS), la Organización Panamericana de la Salud (OPS) y el Fondo de las Naciones Unidas para la Infancia (UNICEF) elaboran sus políticas y programas, entre los cuales se encuentran aquellos destinados a la promoción de la lactancia materna.

Como ejemplo de lo recién mencionado, resulta relevante destacar que desde 1974, año de la realización de la $27^{\mathrm{a}}$ Asamblea Mundial de la Salud, después de reconocer el descenso de la lactancia materna, se exhortó a los países miembros para que adoptaran medidas correctivas. A partir de entonces, diversas reuniones han tenido lugar y de ellas derivaron recomendaciones y normas para lograr aumentar dicha práctica. Al respecto pueden mencionarse el Código Internacional de Comercialización de Sucedáneos de la Leche Materna, adoptado por la 34 a Asamblea Mundial de la Salud en 1981; la Declaración de Fortaleza, en 1985, impulsada por la OMS y la OPS; la Declaración de Innocenti para la protección, promoción y apoyo a la lactancia, en 1990, aprobada en reunión promovida por la OMS y Unicef, al igual que: la Iniciativa Hospital Amigo del Niño, en 1991, en la cual se definieron los "Diez pasos hacia una lactancia exitosa"; y más recientemente la Estrategia Mundial para la alimentación del lactante y del niño pequeño adoptada en 2002 por los Estados miembros de la OMS y la junta ejecutiva del Unicef, así como la Iniciativa para la Humanización de la Asistencia al Nacimiento y la Lactancia, en 2012.

En general, las políticas y programas desarrollados por los organismos internacionales se constituyen en el marco normativo para el diseño de las políticas en materia sanitaria que, como ocurre en México, elaboran los gobiernos federales, estatales, municipales y locales. Cabe añadir que, en el 
diseño de disposiciones o la implementación de programas, generalmente, la participación de las poblaciones no es requerida. De esta manera, en México, a partir de los últimos 27 años, los gobiernos federales de turno han adoptado políticas públicas y programas con el objetivo de lograr el cumplimiento de los acuerdos internacionales para la promoción de la lactancia materna, hecho que ha propiciado modificaciones en diversos códigos legislativos ${ }^{(15)}$.

Muchas de las estrategias encaminadas a promover la salud con temas referidos a la alimentación infantil se incorporan a los programas de carácter social destinados al combate a la pobreza, los cuales, aunque operan en diferentes modalidades, comparten acciones ejecutadas desde diversas dependencias gubernamentales. Entre ellos se encuentran: el Programa Nacional de Solidaridad Social, puesto en marcha en 1988; el Programa de Educación, Salud y Alimentación (PROGRESA) que, en 1997, sustituye al anterior; el Programa de Desarrollo Humano Oportunidades, ejecutado a partir de 2002 y que, en 2014, se sustituye por el Programa Prospera. Actualmente como estrategia de política social opera la Cruzada Nacional Contra el Hambre ${ }^{(29)}$.

A pesar de que en el discurso los programas como Oportunidades se enuncian como integrales, en realidad, en su operación, se mantienen dinámicas bajo las respectivas lógicas sectoriales ${ }^{(30)}$. En su modo de operar se destaca la focalización hacia poblaciones reconocidas por la precariedad en sus condiciones de vida y la asignación de subvenciones. Para incorporar a los usuarios al programa, se los condiciona al cumplimiento de diferentes actividades, asumiendo así la posibilidad de mantener el acercamiento y atención a los beneficiarios. Sin duda, estos programas han recibido muchas críticas por su modo de operar y el uso clientelar que se les ha dado ${ }^{(31)}$.

En México se han destinado enormes recursos para la ejecución de programas encaminados a la promoción de lactancia materna, las acciones emprendidas han resultado ineficaces como lo mostró la Encuesta Nacional de Salud y Nutrición 2012 en la que se registró que $97 \%$ de los infantes llegaron a los seis meses de edad sin lactancia materna, cifras nunca antes encontradas ${ }^{(32)}$. De hecho, ante la tendencia a la baja de esta práctica, se han venido haciendo críticas y recomendaciones a las políticas sanitarias; por ejemplo, desde el año $2003^{(33)}$ se propusieron adecuaciones a las estrategias utilizadas hasta entonces por los programas gubernamentales para su protección y promoción. Más recientemente un grupo de expertos de la Academia Nacional de Medicina ofreció una serie de recomendaciones para una política nacional, de carácter multisectorial, encaminada a mejorar las prácticas de lactancia materna $^{(34)}$. Lo cierto es que resulta evidente la necesidad de cambiar las propuestas para la formulación de políticas destinadas a su promoción, reconociendo que parte de la solución o estancamiento de los problemas se asocia con el pensamiento que direcciona o rige estas acciones gubernamentales.

\section{Anotaciones sobre la aplicación del modelo biomédico en el fomento de la lactancia materna}

Le Bretón reconoce que, desde el famoso texto De humanicorporis fabrica, de Vesalio, “... la representación médica del cuerpo deja de ser solidaria con la imagen integrada del hombre" (35) y que "...la invención del cuerpo en el mundo occidental responde a una triple sustracción: el hombre es separado de él mismo..., separado de los otros..., separado del universo..." ${ }^{\prime 36)}$. En efecto, se reconoce que, orientada por este marco epistémico, la práctica médica ha operado bajo un modelo de atención biomédico seriamente discutido $^{(35,37,38)}$ que según Menéndez, se caracteriza por tener entre otros, los siguientes rasgos estructurales: biologismo, concepción teórica positivista, medicalización, tecnocracia, ahistoricidad y asociabilidad ${ }^{(39,40)}$. Siguiendo a este último autor, para explicar la causalidad del abandono de la lactancia materna y su relación con el modelo médico, hace poco más de 30 años Ysunza ${ }^{(41,42)}$ centró 
su trabajo en cuatro "niveles de análisis", a los que Menéndez ${ }^{(40)}$ considera como dimensiones. A continuación exponemos de forma resumida estos cuatro niveles de análisis propuestos por Ysunza ${ }^{(41,42) \text { : }}$

a. Nivel económico-político: se refiere al control monopólico de la industria de sucedáneos de leche materna bajo el cobijo de las instituciones.

b. Nivel institucional: el autor destaca que, si bien existen políticas que propician la lactancia materna, en la práctica, el discurso se contradice, por ejemplo, en la permisividad para utilizar sucedáneos de leche materna en los hospitales, las prácticas de atención del recién nacido inmediatamente después del parto y su inmediata separación de la madre.

c. Nivel de conocimiento y práctica médica: en los años en los que se realizó el estudio, en las escuelas de medicina no existía como materia prioritaria la de nutrición y los temas relacionados con ella se ofrecían en diferentes cursos apegados al modelo biomédico. Respecto a los conocimientos sobre lactancia materna eran muy limitados e impartidos generalmente en los cursos de pediatría, en los que se enunciaban someramente sus beneficios, en tanto se prestaba mayor atención a la preparación de fórmulas lácteas.

d. Nivel ideológico: se encuentra presente en los tres niveles anteriores y se reconoce como primordial para la operación del modelo médico. Por ejemplo, en el nivel económico-político la presencia ideológica se distinguía en los mensajes publicitarios dirigidos tanto al equipo de salud como a la población. También hace referencia al material didáctico en el que abundaban nociones contradictorias sobre la lactancia que, por un lado, consideraban beneficiosa la leche humana mientras que, por el otro, las múltiples contraindicaciones expresadas de forma generalizada inhibían prácticamente su uso.

Sin duda, distinguir tales dimensiones de análisis resultó afortunado para reconocer diferentes situaciones que estaban obstaculizando la lactancia materna y que aún en la actualidad persisten; sin embargo, es conveniente precisar que existen dinámicas e interacciones que ocurren en y entre las dimensiones propuestas que complejizan aún más la explicación deseada.

A pesar de los cuestionamientos que se han hecho al modelo de atención mencionado, es el pensamiento que orienta la elaboración de políticas y la propia operación de programas del sector salud en México. Parte de la información que sustenta la formulación de estas políticas es la recabada en encuestas elaboradas desde una visión normalizadora, enfocadas en cuantificar las diferentes prácticas de lactancia materna y en seleccionar factores de riesgo o bien determinantes sociales. Para ello, se utilizan "indicadores" de carácter social, como la escolaridad de la madre, lugar de residencia y condición indígena, entre otros, separando los elementos del contexto, sin considerar la multidimensionalidad del proceso. El siguiente texto, que narra los resultados derivados del análisis de la Encuesta Nacional de Salud y Nutrición 2012 es un ejemplo de lo comentado sobre este tema:

En general, los indicadores de lactancia materna muestran un deterioro con respecto a las encuestas anteriores [...] y ha sido en mayor medida en los grupos más vulnerables: en mujeres de medio rural, en la región sur del país, del tercil más bajo de nivel socioeconómico, educación menor a la primaria, aquellas sin acceso a servicios de salud o sin un empleo remunerado; todas estas diferencias son estadísticamente significativas $(p<0,05)$, incluso en hogares beneficiarios del Programa de Desarrollo Humano Oportunidades. ${ }^{(32)}$

Si bien en los años setenta las intervenciones en salud pública se centraban en cambiar comportamientos que enferman por estilos de vida y hábitos saludables ${ }^{(43)}$, la atención colocada en estos últimos sigue estando en boga, como puede constatarse en el 
Informe de evolución histórica de la situación nutricional de la población y los programas de alimentación, nutrición y abasto en México hecho por el Consejo Nacional de Evaluación de la Política Social (CONEVAL) en el cual se sugiere mejorar la estrategia de comunicación educativa promoviendo la adopción de conductas saludables, más que de conocimientos $^{(44)}$, retroalimentando, en el caso de salud, la visión a partir del modelo biomédico.

Para los propósitos del presente trabajo es útil recordar que, desde las incipientes prácticas de la puericultura y la pediatría, dedicadas a la atención de la niñez y por su inevitable vinculación con la alimentación en los niños, la lactancia materna se convirtió también en asunto médico ${ }^{(45)}$. Durante el proceso de medicalización "...los problemas no médicos se vuelven tratados como problemas médicos..." ${ }^{\prime(46)}$, de manera que el corpus sanitario se convierte en el autorizado para mandatar los estilos de vida y comportamientos que deben adoptar las mujeres en torno a las prácticas alimentarias, ejerciendo así una forma de control social sobre las personas y las comunidades ${ }^{(38)}$.

\section{Tensiones y contradicciones en la promoción de la lactancia materna: ante el deber hacer y el hacer}

Como se adelantó al inicio de este texto, durante el trabajo de campo realizado en la comunidad indígena de Cuentepec, en el estado de Morelos, México, se recabaron una serie de testimonios que dan cuenta de cómo el enfoque biomédico se ejerce e impone a través del mismo personal de salud en una situación de interculturalidad. Así, en Cuentepec se observó la imposición de una serie de reglas que las mujeres tenían que cumplir para ser acreedoras de los beneficios de los diferentes programas sociales. Por ejemplo, las mujeres "beneficiarias" del programa Oportunidades, durante su etapa de embarazo y después del nacimiento de su hijo, tenían el compromiso de asistir a las pláticas impartidas por el personal de salud (enfermeras y promotores de salud) en

las que se les indicaba lo que "debían ser y hacer" utilizando para ello instrucciones muy alejadas de sus códigos semánticos y de su práctica cotidiana ${ }^{(43,47)}$. Bajo esta situación, las tensiones que se percibieron en el testimonio del personal de salud fueron claras: ...me siento rara al platicar con ellas,
hablan náhuatl y son muy diferentes...
(Personal de enfermería pasante del
Centro de Salud, Entrevista A, noviembre
de 2008)

...ni caso nos hacen... no obedecen...

(Personal promotor del programa Oportunidades, noviembre de 2008)

Asimismo, para recibir los beneficios del programa Oportunidades, las mujeres embarazadas tenían, como condición, que su parto fuera atendido por personal sanitario acreditado, ya fuera en servicio particular o público. Siguiendo a Le Breton ${ }^{(35)}$ las mujeres eran separadas de su propio cuerpo, dejaban de ser personas para convertirse en objetos -cuerpos preñados proveedores de leche-; personas separadas de su familia o seres queridos, rompiendo con ello las redes de apoyo tradicionales estructuradas en su círculo familiar para el momento del parto y apartadas de su contexto cultural. Como en el centro de salud de Cuentepec no se atendían partos $y$, para parir, las mujeres se trasladaban al Hospital Amigo del Niño y de la Madre en la localidad de Tetecala, comunidad cercana, lo que suscitaba innecesariamente el distanciamiento de sus tradiciones, pues dejaban de ser atendidas por las parteras de la propia comunidad, propiciando dudas e inconformidades. Como se observa en los siguientes testimonios:

\footnotetext{
...antes venía la partera a la casa... nosotras los tuvimos con partera... y sí tuvimos leche [...] te sientes mejor con partera [...] antes aquí había dos parteras... ahora aquí en Cuentepec ya no hay parteras, quien sabe por qué... (Madre lactante joven. Entrevista 16, noviembre 2008)
} 
...los primeros fueron con la partera. Estaba mejor así. Con el último ya fui al Hospital... fue muy diferente... (Madre lactante joven. Entrevista 18, diciembre de 2008)

Llevar a cabo el parto en clínicas u hospitales, implica que las madres sean separadas de su bebé, lo cual imposibilita el amamantamiento inmediato y se dificulta el inicio temprano de la lactancia materna. Además, en estos lugares no pueden recurrir a sus prácticas tradicionales para propiciar la secreción láctea ya que el personal sanitario devalúa tales conocimientos por no corresponder con los procedimientos reconocidos por la biomedicina. Una persona que ejerció como partera antes de que llegaran los servicios de salud recordó los beneficios de atender el parto según sus tradiciones:

...cuando todavía no nace la placenta... [amamantar] le ayuda como a estimular... a extraer la placenta, ...antes de cortarle... hay que darle el niño a la mamá... le ayuda a sacar la placenta [...] y también para que se conozcan luego, luego [...] después de limpiarlos... rápido...a darle [el pecho]..., bueno, aunque unas prefieren dormir, otras prefieren comer, otras prefieren llorar... (Personal promotor de Salud, antes partera, noviembre de 2008)

Las mujeres de Cuentepec tienen plena convicción de amamantar a su hijo y valoran los conocimientos transmitidos por las madres y las abuelas de generación en generación, sobre el conjunto de conocimientos y actividades para practicarla, reconocidos como la cultura de la lactancia materna y que hasta la fecha perduran.

...así dice mi mamá que le dé...porque así me daba ella a mí... (Madre lactante joven. Entrevista 31, marzo de 2009)

...así es aquí... aquí todas dan pecho... (Madre lactante joven. Entrevista 36, marzo de 2009)
Parte de los saberes tradicionales para estimular la producción de leche o "bajada de la leche", utilizan la "jarilla", nombre que se le da al atado de hojas de maíz que contiene la hierba "atzomali" -nombre náhuatlremojada en alcohol, el cual es calentado previamente en el comal para ser colocado sobre los senos.

En contraste, desde el modelo biomédico, además de la verticalidad en el diseño de los programas impuestos, fue claro el empoderamiento del saber médico sobre el saber de la comunidad, el cual no era tomado en cuenta, además de ser paradójicamente contradictorio con los procedimientos acordados por las más altas instancias sanitarias. Esta situación se observa claramente en el testimonio de un médico con puesto directivo en el hospital Amigo del Niño y de la Madre de Tetecala, el cual indicaba rutinariamente el uso de fórmula en los casos en que el tiempo de atención posparto se prolongaba por la inexperiencia del médico interno:

...si ha pasado más de media hora, sí, le damos una toma de leche para no esperar tanto tiempo, porque el niño no se puede quedar en ayuno... le da hipoglucemia, convulsiona y se muere... así es que necesitamos siempre prevenir ¿no? Tenemos a la mano la leche en caso de necesidad. (Médico del Hospital Amigo del Niño y de la Madre de Tetecala, abril de 2009)

...tenemos las fórmulas especiales que ya están licitadas y disponibles en almacén. Cuando iniciamos la vía oral es entonces cuando usamos las fórmulas para prematuro, primero toleramos con la fórmula, si aguantan, cambiamos al seno materno. (Médico del Hospital Amigo del Niño y de la Madre de Tetecala, abril de 2009)

Desde su perspectiva biologicista, el médico estaba convencido de que las recomendaciones que les transmitían a las madres durante las consultas en las que revisaba a los recién nacidos eran la única razón para que 
ellas dieran el pecho a sus bebés sin tomar en cuenta las prácticas culturales de lactancia de la comunidad de origen. En sus sugerencias para ejercer la lactancia a libre demanda era clara su postura medicalizada:

\section{...si les metemos mucho en la cabeza que es como una vacuna, que cada vez que dan [leche materna] ...es como vacunar al niño...entonces se van con esa idea y se lo están pegando a cada rato... (Médico del Hospital Amigo del Niño y de la Madre de Tetecala, abril de 2009)}

Uno de los objetivos primordiales de los programas dirigidos a la promoción de la salud materna e infantil es el de ofrecer apoyos e información para fomentar la lactancia materna. Un aspecto fundamental en este proceso es la preparación y capacitación que debe tener el personal de salud involucrado para ofrecer el apoyo y la información necesaria. Como ocurre en otros lugares, la preparación sobre el tema que reciben los médicos y, en general, el personal de salud durante su formación profesional, es muy escasa ${ }^{(48,49,50,51)}$. Lo anterior se ilustra a través del testimonio aportado por el personal médico responsable del centro de salud de Cuentepec, quien comentó haber estudiado en una universidad de reconocido prestigio nacional en donde cursó solamente un módulo docente con duración de un año llamado Parto y puerperio y precisó que "... dentro de la materia... se dedicó una sesión completa al tema..." que abarcó, entre otros, la lactancia materna y la artificial. Así, aunque los contenidos teóricos y prácticos sobre lactancia humana habían sido someramente abordados, le parecían suficientes, comentario muy similar al del médico de Tetecala.

Por otra parte, resulta importante señalar que la estrategia de comunicación educativa para operar los programas mencionados, desde el nivel central al local, sigue el modelo de capacitación en "cascada", apegado al modelo educativo basado en la enseñanza cuya eficacia ha sido cuestionada(50,52). Bajo esta lógica de pensamiento al personal de salud se le instruye para reproducir los conocimientos o mensajes diseñados como política pública ${ }^{(42)}$ en la que se asume que el mensaje será trasmitido tal y como se generó desde el nivel central. Lo cierto es que los mensajes en el nivel local llegan con muchas imprecisiones y errores, trastocados por las subjetividades de las personas que intervienen en el proceso. Por ejemplo, se desalentaba la continuación de la lactancia materna expresando reiteradamente opiniones como:

$$
\begin{aligned}
& \text {...la leche materna se va convirtiendo } \\
& \text { en agua... (Personal de enfermería } \\
& \text { pasante del Centro de Salud. Entrevista } \\
& \text { B, abril 2009) }
\end{aligned}
$$

Respecto a la duración de la lactancia, se recomendaban las fórmulas lácteas a partir de los seis meses:

...No, obviamente la leche [materna], la leche es importante para el niño, pero les recomendamos nosotros las fórmulas, ahí si ya empezamos a meter fórmulas de segunda etapa. A partir de los seis meses. (Médico del Hospital Amigo del Niño y de la Madre de Tetecala, abril 2009)

Ante los testimonios arriba expuestos, se observa que la mayor confianza del personal de salud está en los sucedáneos de leche humana, más que en la propia práctica de la lactancia materna, por lo que la consiguiente recomendación del personal médico hacia las madres, es para fomentar el uso de fórmulas de diferentes marcas con las que el personal de salud estaba más familiarizado en su manejo.

Las afirmaciones del personal de salud muestran desconocimiento de las recomendaciones aceptadas internacionalmente; sin embargo, queda de manifiesto la relación asimétrica y de subordinación que ocurre entre el médico y la paciente, propio del modelo de atención biomédico, que conduce a la interposición de barreras que impide una comunicación clara y horizontal entre estos dos actores ${ }^{(47)}$. Según los testimonios, los médicos no solo desaconsejaban la lactancia materna después de los seis meses, sino que desalentaban y desaprobaban francamente 
su continuación ${ }^{(53,54,55,56)}$, devaluando las tradiciones y prácticas de alimentación y crianza "diferentes" a la interpretación de sus saberes, al considerarlas incorrectas o francamente peligrosas para la salud. Para reforzar estas prohibiciones los médicos vinculaban la "resistencia e indisciplina maternas" con enfermedades en la madre y el niño.

La narrativa reiterada de las madres fue sobre las indicaciones o mandatos recibidos de todos los médicos en Cuentepec respecto al destete de los bebés, fijándolo cuando mucho a los seis meses, argumentando sobre las desventajas y advertencias sobre las supuestas consecuencias de prolongar el amamantamiento.

...dicen que... después del año ya no les sirve [a los bebés] la leche, que es como si estuvieran tomando pura agua... (Madre lactante joven. Entrevista 24, diciembre 2008)

...aquí nos dicen que ya no es leche, que es como mi sangre y que le hace daño a mi bebé y a mi si le doy... (Madre lactante joven. Reunión focal, marzo 2009)

Lo anterior propició que, respecto a la edad recomendada para iniciar la alimentación complementaria y el posterior destete, las madres jóvenes se refirieran en las entrevistas al "deber hacer" pensando que fuera acorde con el mensaje recibido del personal sanitario. Así, colocadas en un nivel de subordinación, manifestaron que consideraban que los médicos eran los que sabían más sobre lactancia materna:

...Pues yo creo que los doctores, yo digo que todos saben, porque estudiaron más, saben como qué enfermedades te pueden afectar o qué peso debe tener el bebé... (Madre lactante joven. Entrevista 11, noviembre de 2008)

Sin embargo, algunas se atrevieron a reconocer la valía de sus conocimientos tradicionales: ...mmm..., no sé, las mamás saben más porque ya dieron pecho... ¿no? (Madre joven. Entrevista 8, noviembre 2008)

...las mamás [saben más de lactancia] las que dan las pláticas no tienen experiencia... (Madre lactante joven. Entrevista 16, noviembre 2008)

...sí, yo creo que... mmm... nosotras sabemos de eso... (Madre lactante joven. Entrevista 24, diciembre de 2008)

A pesar de que el "deber ser" médico era que había que destetar a los niños a los seis meses, los resultados de la investigación permitieron observar que las prácticas de amamantamiento seguían siendo predominantemente tradicionales, ya que varias mujeres que asistieron a la reunión focal, declararon que ellas continuaban amamantando de acuerdo a las costumbres ancestrales y practicadas enseñadas por sus madres y abuelas. Señalaron la práctica de una lactancia que podía ser prolongada, hasta que los niños tenían entre tres y cinco años de edad como se muestra en los siguientes testimonios:

...ya estaba yo grande...y me acuerdo de cuando mi mamá iba a recogerme a la escuela, corría yo a pegarme a la chiche... (Madre lactante joven. Entrevista 10, noviembre de 2008).

No quiere dejarlo [...] chilla mucho y me pide [...] mejor le sigo dando [...] antes nos daban hasta que estábamos grandes... (Mujeres lactantes jóvenes. Reunión focal, marzo de 2009)

\section{DISCUSIÓN}

En la práctica, la atención materno-infantil básicamente se opera en los servicios de primer nivel, que se encuentran distribuidos en muy diversas regiones del territorio 
mexicano, país caracterizado por su multiculturalismo ${ }^{(57)}$. A través de la información recabada en el trabajo de campo, se confirmó que el personal sanitario reconoció no tener mayor preparación sobre el tema de lactancia materna, deficiencia ya registrada por otros autores desde hace años en México y otros países $^{(41,48,49,53,58)}$. Es así que las recomendaciones para mejorar la salud y propiciar el cambio en los patrones de conducta y su implementación institucional en poblaciones diversas culturalmente, dependen de la apreciación y valoración del propio personal sanitario que al ejercer estas funciones, sin contar con una formación teórica y práctica para comprender la pluralidad biocultural, asume su papel de "educador" desde su percepción, cultura y subjetividad a partir de sus saberes informales adquiridos dentro de sus propios contextos culturales occidentales, propagando mensajes contradictorios, mal informados y desalentadores para continuar con la lactancia materna más allá de los seis meses, acompañada con la introducción de nuevos alimentos.

Ante esta diversidad cultural, el modelo biomédico, que empodera al saber médico por sobre el de la comunidad, limita seriamente al personal sanitario para conocer, aceptar y reconocer las diferentes prácticas culturales de crianza, incluidas la de alimentación infantil. De la misma manera, facilita la persistencia de una apreciación devaluada de tales prácticas, juzgándolas como incorrectas o dañinas, además de invisibilizar a los principales actores que intervienen -madre/hijoquienes, en su vivir diario en un contexto temporal, sociocultural e históricamente determinado, piensan, significan, representan y actúan de forma única; como señala Aisenson ${ }^{(59)}$ : se trata de cuerpos-persona. A su vez, dicho modelo, no toma en consideración que muchas comunidades con características rurales, todavía mantienen fuerte arraigo en sus patrones bio-socio-culturales de alimentación infantil y de crianza ${ }^{(16,56)}$ que han coadyuvado a una visión particular del mundo y de su propia comunidad; patrones gestados mucho antes del arribo de programas gubernamentales de apoyo.
En poblaciones como Cuentepec, con fuerte arraigo en su cultura y con una organización social que mantiene a las mujeres unidas por lazos familiares, las actitudes de apoyo mutuo se refuerzan en el tema de la crianza y la lactancia, como lo ha reconocido Massó( ${ }^{(60)}$. Al respecto, se observó que la desaprobación de los servicios médicos no ha promovido el abandono de esta práctica en la comunidad, por el contrario, de manera involuntaria ha coadyuvado a una mayor unidad y alianza entre las mujeres para compartir convicciones, costumbres, tiempos y formas para atender y alimentar a sus hijos.

Algunos trabajos han reportado en otras comunidades rurales, mensajes provenientes del personal sanitario similares a los registrados en Cuentepec ${ }^{(53,54,55,56,61)}$, los cuales, de alguna manera asocian el abandono de la lactancia materna con las recomendaciones recibidas por dicho personal. Sin duda es necesario realizar más trabajos de corte antropológico para corroborar el impacto de la información contradictoria y subjetiva que se brinda en estas regiones y explorar si estas indicaciones pueden tener un efecto negativo y desalentador de esta práctica. En tanto, a manera de hipótesis, suponemos que cuando las mujeres carecen de cohesión social, esta condición propicia su vulnerabilidad ante la penetración de comportamientos de crianza ajenos a su cultura y cosmovisión lo que obstaculiza la transmisión de sus saberes tradicionales para atender la crianza y la lactancia materna y asumir como necesaria la utilización de sucedáneos de la leche materna.

Es deseable que antes de introducir programas para cambiar comportamientos y estilos de vida, se haga un diagnóstico cultural previo, se dialogue con la comunidad y se analice su pertinencia. Se pudo constatar que, en esta población, dichos programas resultaban innecesarios pues la gran mayoría de las mujeres entrevistadas se sentían seguras de sus conocimientos y seguían sus estrategias para iniciar y continuar con sus lactancias de manera muy exitosa.

Las situaciones que se han discutido hasta aquí y que generan tensión y contradicción en las madres lactantes, pareciera radicar 
únicamente en un asunto que compete a las instancias responsables de la formación profesional y capacitación de los trabajadores de la salud. Al respecto, se puede citar el trabajo de investigación realizado por Caicedo et al. ${ }^{(52)}$, en el que encontraron que las mujeres lactantes y sus familiares tuvieron contradicciones y desencuentros con el personal de salud que las atendió, lo cual motivó la necesidad de generar una práctica educativa diferente. Así, acertadamente propusieron una nueva estrategia educativa acorde con la complejidad del proceso de la lactancia materna con el propósito de "...establecer una relación con el otro incluyente, respetuosa y equitativa...", la cual se apoyó en "... los postulados del interaccionismo social de Vygotski, la teoría de la complejidad de Morin y la práctica reflexiva de Schön"(52). Sin duda, es una propuesta pedagógica necesaria, pero dadas las circunstancias del contexto teóricopolítico prevalecientes en el sector sanitario, se requiere trascender el modelo biomédico imperante para que pueda ser adoptada por los programas gubernamentales.

Hace poco más de 30 años ya se había demostrado la disminución en la práctica de la lactancia materna en México, especialmente en las zonas urbanas pero, además, en aquel entonces, se puso atención en que el problema también existía en las zonas rurales $^{(41)}$ y se hicieron planteamientos teóricos sobre su causalidad ${ }^{(42)}$. Desafortunadamente, la mayoría de las veces, las observaciones de corte sociocultural son poco atendidas por los responsables de elaborar e instrumentar las políticas sanitarias por lo que, a pesar de los pronunciamientos y directrices encaminados a la protección de prácticas sanas de alimentación infantil, las acciones pro lactancia materna han sido poco eficaces. Por ejemplo, entre otros procesos, el efecto de algunas políticas de apoyo evaluadas por el CONEVAL ${ }^{(44)}$ señalaron una diferencia de 2,3 meses menos en la duración de la lactancia materna entre las mujeres participantes en el programa Oportunidades y las no inscritas en dicho programa.

Si bien la intención central de las políticas públicas destinadas a promover la lactancia materna pretende mejorar la salud y nutrición de los niños, su elaboración desde las cúpulas sectoriales y encuadrada en el modelo biomédico, les confiere una perspectiva muy distante de las realidades locales, lo que las ha llevado a resultados poco exitosos e incluso negativos, que no solo no promueven la lactancia materna, sino que propician prácticas que atentan contra la cultura propia de las comunidades. Ante estas situaciones ¿qué rumbo tomar?

Según Morin, "la acción política se ha basado siempre, implícita o explícitamente, en una concepción del mundo, del hombre, de la sociedad y de la historia, es decir, en un pensamiento" ${ }^{\prime(62)}$ o paradigma, entendido como tal, el sistema de creencias, categorías, normas y valores fundamentales a partir de los cuales un grupo humano produce y reproduce conocimiento, orienta su acción social e induce prácticas que generan consensos para explicar el mundo ${ }^{(63)}$. A lo largo de ese texto se han presentado las limitaciones que impone el modelo biomédico implícito en el diseño de las políticas, programas y en su ejecución para promover la lactancia materna; en consecuencia, es urgente un cambio en el modelo de acción de dicho paradigma, teniendo claro que "no hay reforma política sin reforma del pensamiento político, el cual supone una reforma del pensamiento mismo..." ${ }^{\prime(62)}$, es decir, un cambio de paradigma.

Como ocurre en la mayoría de los campos disciplinarios, el pensamiento médico occidental ordena su saber a partir de lo que Edgar Morin ${ }^{(64,65)}$ define como el paradigma del pensamiento simple, o lo que Vilar Ilama vieja racionalidad ${ }^{(66)}$. Se trata de una lógica que separa los componentes que forman parte de un todo, en otras palabras, de un sistema, con lo cual dejan de manifestarse los hechos que tienen lugar durante las interacciones entre sus diferentes componentes. Esta lógica también tiende a unificar de forma abstracta los componentes que son diversos y propicia pensar unidimensionalmente, además de ser ultrajerárquica. En contraste, se propone optar por una nueva racionalidad ${ }^{(66)}$ que Morin $^{(64,65)}$ reconoce como paradigma del 
pensamiento complejo; lógica que se caracteriza por distinguir componentes que integran sistemas pero sin separarlos ni desarticular el propio sistema; además, es capaz de conjugar los componentes diversos que lo integran, sin invisibilizarlos o jerarquizarlos y facilita pensar la multidimensionalidad de las realidades.

Antes de abordar de manera general algunas situaciones que muestran cómo es comprendida la lactancia materna desde la lógica del pensamiento simple, diremos que es inusual que se la reconozca como un sistema multidimensional (biopsico-socio-cultural, determinado históricamente ${ }^{(14,15,16,56)}$, en el que tienen lugar interacciones que ocurren entre las personas, no solo entre la conocida díada madre/hijo, sino también entre otros integrantes de la familia, amigos o miembros de la comunidad, así como con el personal sanitario; estas interacciones no ocurren en contextos homogéneos, sea desde la perspectiva biológica, sociocultural o económica.

\section{Propuesta para la comprensión y atención de la promoción de la lactancia materna como proceso bio- psico-socio-cultural}

A lo largo del texto se han formulado críticas a acciones emprendidas que se corresponden con el modelo biomédico imperante y que se consideran apegadas a los principios rectores del paradigma del pensamiento simple. A manera de ejemplo, a continuación, se presentan dos escenarios en torno a la lactancia materna, en los que prevalece la racionalidad del pensamiento simple, y se propone una nueva mirada que pone de manifiesto la racionalidad compleja.

El primero de ellos se refiere a las intervenciones gubernamentales para la promoción de la lactancia materna, en los que se distingue la posición ultrajerárquica, la lógica que unifica lo que es diverso y el pensar unidimensionalmente. Esto ocurre a partir de la elaboración de las políticas, programas y proyectos de intervención en las comunidades $^{(67)}$, los cuales generalmente se diseñan por expertos desde las cúpulas, sin incorporar a las personas a las que se dirigen, y se programan acciones predeterminadas $y$ prácticamente idénticas para todo tipo de población. En el caso de la lactancia materna, los programas van dirigidos a las mujeres que pronto iniciarán o ya han iniciado su lactancia, es decir a mujeres-madres que comparten un mismo estadio de vida por lo que suponen se trata de una unidad homogénea, sin advertir que en realidad no lo es, pues hay diversidad sociocultural entre ellas. En efecto, ni en la formulación de los programas ni en su ejecución se consideran los saberes, sistemas de creencias y prácticas culturales propias de la cultura a la que pertenecen las pacientes (mujeres embarazadas y lactantes) que serán intervenidas. Ello propicia, entre otros, falta de comunicación, contradicciones y finalmente el fracaso de los objetivos de las acciones.

El segundo escenario que se desea ejemplificar, alude a los procedimientos para conocer los comportamientos en torno a la práctica de la lactancia materna y la causalidad de su abandono, para lo cual se generan investigaciones y encuestas, separando, desde su inicio, los componentes que integran el sistema y se opta por estudiar los factores (aislados unos de otros) que inciden en ella, lo que impide observar otras realidades. Por ejemplo, una madre recién parida puede ser apoyada por la red femenina de familiares o amistades, lo que propicia confianza, seguridad, aceptación, entre otras, y facilita el inicio y continuación de la lactancia materna; en contraste, con frecuencia ocurren desencuentros entre las mujeres lactantes y el personal de salud que tiene el poder de orientar las acciones, porque es poseedor del conocimiento correcto y desconoce o menosprecia los saberes de la cultura local a la que ellas pertenecen. Esta situación suele generar estados de frustración, confusión o franco malestar, que suelen ser algunas de las causas de no iniciar o suspender la lactancia materna antes del tercer mes, circunstancias que no son observadas y en consecuencia 
no se consideran como "factores" en la explicación, ni para modificar las normas de operación de los programas.

Como señalan tanto Almeida-Filho como Samaja, y concordando con Boaventura Santos es necesaria la radical transformación “...del sistema de formación de los sujetos de la ciencia, en contexto de un nuevo paradigma, capaz de construir síntesis y operar tránsitos no solo interdisciplina, sino interparadigmas y, aún más, entre los saberes de la vida y los conocimientos de la ciencia"(68). Cuando el pensamiento médico occidental transite del paradigma del pensamiento simple al de la complejidad, se facilitará mirar, pensar y comprender la multidimensionalidad de los procesos vinculados con la salud, en nuestro caso, de la lactancia ma-

\section{AGRADECIMIENTOS}

Agradecemos la colaboración de todas las personas entrevistadas, tanto el personal de los servicios de salud como de las mujeres lactantes, madres y abuelas de Cuentepec, quienes compartieron sus saberes, opiniones y emociones. Agradecemos a la Universidad Nacional Autónoma de México la cual, a través de la Dirección General de Personal Académico otorgó el financiamiento para la obtención de la información mediante la beca Programa de Apoyo a Proyectos de Investigación e Innovación Tecnológica (PAPIIT) IN-404508 otorgada al Proyecto "Enfoque antropológico de los codeterminantes del cambio en la condición nutricia de los habitantes de Cuentepec, Morelos" y del cual se derivó parte de la información que se presenta. De la misma manera reconocemos a las personas que enriquecieron este trabajo con sus valiosos comentarios.

\section{REFERENCIAS BIBLIOGRÁFICAS}

1. Tanner JM. Foetus into man: physical growth from conception to maturity. Cambridge Massachusetts: Harvard University Press; 1978.

2. Dobbing J, Sands J. Comparative aspects of the brain growth spurt. Early Human Development. 1979;311:79-83.

3. Narvaez D, Panksepp J, Schore AN, Gleason TR. The value of using an evolutionary framework for gauging children's well-being. En: Narvaez D, Panksepp J., Schore AN, Gleason TR (eds.). Evo- terna, teniendo presente su historicidad y temporalidades. Entonces el acercamiento a las realidades dejará de ser vertical, tecnocrático, reducido, jerárquico, asocial, ahistórico ${ }^{(39,40)}$. El pensamiento médico será el que reconozca, entre otros, la diversidad en la unidad de los contextos socioculturales en los que tiene lugar la vida de las personas, y reconozca la valía de dicha diversidad; el que considere la integralidad de las personas (mente-cuerpo) sin que sean cosificadas; el que apruebe entablar un diálogo respetuoso, participativo y horizontal con las personas a las que dirige sus servicios, lo que le permitirá un acercamiento mayor a la comprensión de los procesos bio-psico-socio-culturales propios del ser humano, como es la lactancia materna.

lution, early experience and human development: from research to practice and policy. Oxford: Oxford University Press; 2012.

4. Victora CG, Bahl R, Barros AJ, França GV, Horton S, Krasevec J, Murch S, Sankar MJ, Walker N, Rollins C, The Lancet Breastfeeding Series Group. Breastfeeding in the 21st century: epidemiology, mechanisms, and lifelong effect. The Lancet. 2016; 387(10017): 475-490.

5. Fouts HN, Hewlett BS, Lamb ME. A biocultural approach to breastfeeding interactions in Central Africa. American Anthropologist. 2012;114(1):123136. Doi: 10.1111/j.1548-1433.2011.01401.x.

6. Torre Medina-Mora P, Salas Valenzuela $M$. Una revisión bibliométrica de la alimentación infantil en México. Gaceta Médica de México. 2013;149(1):53-60.

7. Ballard O, Morrow AL. Human milk composition: nutrients and bioactive factors. Pediatric Clinics of North America. 2013;60(1):49-74.

8. Kramer MS, Aboud F, Mironova E, Vanilovich I, Platt RW, Matush L, Igumnov S, Fombonne E, Bogdanovich N, Ducruet T, Collet J, Chalmers B, Hodnett E, Davidovsky S, Skugarevsky O, Trofimovich O, Kozlova L, Shapiro S, Promotion of Breastfeeding Intervention Trial (PROBIT) Study Group. Breastfeeding and child cognitive development new evidence from a large randomized trial. Archives of General Psychiatry. 2008;65(5):578584. Doi:10.1001/archpsyc.65.5.578. 
9. Rollins NC. Bhandari N, Hajeebhoy N, Horton, S, Lutter CK, Martines JC, Piwoz EG, Richter LM, Victora CG, The Lancet Breastfeeding Series Group. Why invest, and what it will take to improve breastfeeding practices? The Lancet. 2016;387(10017):491504.

10. Colchero MA, Contreras-Loya D, Lopez-Gatell $\mathrm{H}$, de Cosío TG. The costs of inadequate breastfeeding of infants in Mexico. The American Journal of Clinical Nutrition. 2015;101(3):579-586.

11. Organización Mundial de la Salud. Estrategia mundial para la alimentación del lactante y del niño pequeño [Internet]. Ginebra: OMS; 2003 [citado 10 dic 2016]. Disponible en: https://goo.gl/4Woppp.

12. González de Cosío T, Escobar-Zaragoza L, González-Castell L D, Rivera-Dommarco JA. Prácticas de alimentación infantil y deterioro de la lactancia materna en México. Salud Pública de México. 2013;55(Suppl 2):S170-S179.

13. Silva Ayçaguer LC. A statistical ceremony to identify risk factors. Salud Colectiva. 2005; 1(3):309322.

14. Salas Valenzuela M, Torre P. Datos recientes sobre alimentación infantil en México: omisión de aspectos socioculturales. 1er Congreso Latinoamericano de Antropología; 11-15 jul 2005, Facultad de Humanidades y Artes, Rosario, Argentina.

15. Torre Medina-Mora P, Salas Valenzuela M. La alimentación infantil en el siglo XX: Un recuento crítico en torno a leyes, programas y acciones públicas en salud en México. En: Chapela Mendoza MC, Contreras Garfias ME, (coords.). La salud en México: Pensar el futuro de México. México: UAM-Xochimilco; 2011.

16. Trujillo, JI, Peñaranda Correa F, Otálvaro Orrego JC, Bastidas Acevedo M, Torres Ospina JN. La lactancia materna como un fenómeno singular y sociocultural surcado por diferencias y tensiones. Archivos Venezolanos de Puericultura y Pediatría. 2015;78(2):45-51.

17. Dunbar RI, Shultz S. Evolution in the social brain. Science. 2007;317(5843):1344-1347.

18. Morin E. El paradigma perdido: Ensayo de bioantropología. Barcelona: Editorial Kairós; 1974.

19. Fuentes A. The extended evolutionary synthesis, ethnography, and the human niche: toward an integrated anthropology. Current Anthropology. 2016;57(Suppl 13):S13-S26.
20. Jelliffe DB, Jelliffe EP. Human milk in the modern world. Oxford London: Oxford University Press; 1978.

21. Stuart-Macadam P, Dettwyler KA, (eds.). Breastfeeding: biocultural perspectives. New York: Aldine de Gruyter; 1995.

22. Menéndez EL. Epidemiología sociocultural: propuestas y posibilidades. Región y Sociedad. 2008;20(spe 2):5-50.

23. Hernández-González MG. Leche, vida y tradición: Lactancia materna en Cuentepec, Morelos. [Tesis de Maestría en Antropología]. México: Facultad Filosofía y Letras, Instituto de Investigaciones Antropológicas, Universidad Nacional Autónoma de México; 2012.

24. Secretaría de Gobernación. Índice de marginación por localidad [Internet]. 2010 [citado 7 junio 2017]. Disponible en: https://goo.gl/ hRUF8o.

25. Velásquez Gavilanes R. Hacia una nueva definición del concepto "política pública". Desafíos. 2009;20:149-187.

26. Tapia M, Campillo B, Cruickshank S, Morales G. Manual de incidencia en políticas públicas. México: Alternativas y Capacidades; 2010.

27. Parsons W. Políticas públicas: una introducción a la teoría y la práctica del análisis de políticas públicas. México: FLACSO México; 2013.

28. López Arellano O, Rivera Márquez JA, Blanco Gil J. Determinantes sociales y salud-enfermedad en México: Cambios, rupturas y perspectivas. En: Chapela Mendoza MC, Contreras Garfias ME, (coords.). La salud en México: Pensar el futuro de México. Colección conmemorativa de las revoluciones centenarias. México: UAM-Xochimilco; 2011

29. Secretaría de Desarrollo Social. Cruzada Nacional Contra el Hambre [Internet] 2016; México: SEDESOL Gob. Méx [citado 15 ene 2017]. Disponible en: https://goo.gl/NvYh2H.

30. Ramos Rodríguez RM. Reflexiones sobre la intersectorialidad como estrategia para la salud: conocimientos complejos para realidades complejas. Salud Problema, Nueva Época. 2001;6(1011):67-72.

31. Boltvinik J. Políticas focalizadas de combate a la pobreza en México: El Progresa/Oportunidades. En: Boltvinik J, Demián A, (coords.). La pobreza en México y el mundo: Realidades y desafíos. México: Siglo XXI Editores; 2004. p. 315-347. 
32. González de Cosío T, Escobar-Zaragoza L, González-Castell LD, Rivera-Dommarco JA. Prácticas de alimentación infantil y deterioro de la lactancia materna en México. Salud Pública de México. 2013;55(Suppl 2):S170-S179.

33. González de Cossío T, Moreno Macías H, Rivera JA, Villalpando S, Shamah Levy T, Monterrubio EA, Hernández Garduño A. Breast-feeding practices in Mexico: Results from the Second $\mathrm{Na}$ tional Nutrition Survey, 1999. Salud Pública de México. 2003;45(Suppl 4):S477-S489.

34. González de Cosío T, Hernández-Cordero S, Rivera-Dommarco J, Hernández Ávila M. Recomendaciones para una política nacional de promoción de la lactancia materna en México: postura de la Academia Nacional de Medicina. Salud Pública de México. 2017;59(1):106-113.

35. Le Breton D, Gómez MM, Borrillo D. Lo imaginario del cuerpo en la tecnociencia. Reis: Revista Española de Investigaciones Sociológicas. 1994;(68):197-210.

36. Le Breton D. Antropología del cuerpo y modernidad. Buenos Aires: Ediciones Nueva Visión; 1990.

37. Foucault M. Historia de la medicalización. Educación Médica y Salud. 1977;11(1):3-25.

38. Conrad P. The medicalization of society: On the transformation of human conditions into treatable disorders. Baltimore: The Johns Hopkins University Press; 2008.

39. Menéndez EL. Hacia una práctica médica alternativa: Hegemonía y autoatención (gestión) en salud. México DF: CIESAS; 1982.

40. Menéndez EL. El Modelo Médico y la Salud de los Trabajadores. Salud Colectiva. 2005;1(1):9-32.

41. Ysunza A. The decline of breast fedding in Mexico: an example of medical-academic deformation. En: Jelliffe DB, Jellife EF, (eds.). Advances in International Maternal and Child Health. Vol 4. Oxford: Oxford University Press; 1984.

42. Ysunza A. El abandono de la lactancia materna en México II: Su causalidad. Revista de Investigación Clínica. 1986;38(Supl):S97-S102.

43. Davini MC, Nervi L, Roschke MA. Capacitación del personal de los servicios de salud: Proyectos relacionados con los procesos de reforma sectorial. Quito: OPS; 2002.

44. Consejo Nacional de Evaluación de la Política Social. Informe de evolución histórica de la situación nutricional de la población y los programas de alimentación, nutrición y abasto en México [Internet]. 2009 [citado 10 ene 2015]. Disponible en: https://goo.gl/nGFoSP.

45. Illich I. Némesis médica: la expropiación de la salud. Barcelona: Barral Editores; 1975.

46. Conrad P. Medicalization and social control. Annual Review of Sociology.1992;18(1):209-232.

47. Marton B, Echazú G. La violencia simbólica en la consulta médica: la naturalización de la díada madre-hijo y la promoción compulsiva de la lactancia materna. Sexualidad, Salud y Sociedad-Revista Latinoamericana. 2010;5:119-143.

48. Pound CM, Williams K, Grenon R, Aglipay M, Plint AC. Breastfeeding knowledge, confidence, beliefs, and attitudes of Canadian physicians. Journal of Human Lactation. 2014;30(3):298-309.

49. Sigman-Grant $M$, Kim $Y$. Breastfeeding knowledge and attitudes of Nevada health care professionals remain virtually unchanged over 10 years. Journal of Human Lactation. 2016;32(2):350-354.

50. Barr RB, Tagg J. From teaching to learningA new paradigm for undergraduate education. Change: The Magazine of Higher Learning. 1995;27(6):12-26.

51. Salas Valenzuela $M$, Torre Medina-Mora $P$, Meza Segura C. Alimentación infantil: una reflexión en torno a los programas de estudio de enfermería en la Ciudad de México. Salud Colectiva. 2014;10(2):185-199.

52. Caicedo Martínez NS, Carrillo Pineda $M$, Gómez Dávila JG, Ocampo Rivera D, Zea Bustamante LE. Educación para la lactancia materna: una propuesta pedagógica. Medellín: Universidad de Antioquia; 2017.

53. Potter JE, Dávila OM, Hernández D. Opinión del personal de salud sobre el patrón de lactancia en el área rural de México. Salud Pública de México. 1991;33(1):18-28.

54. Vandale-Toney S, Rivera-Pasquel ME, Kageyama-Escobar M, Tirado-Gómez LL, López-Cervantes M. Lactancia materna, destete y ablactación: una encuesta en comunidades rurales de México. Salud Pública de México. 1997;39(5):412-419.

55. Guerrero ML, Morrow RC, Calva J), Ortega-GaIlegos H, Weller SC, Ruiz-Palacios G, Morrow AL. Rapid ethnographic assessment of breastfeeding practices in periurban Mexico City. Bulletin of the World Health Organization. 1999;77(4):323-330. 
56. Reyes Gutiérrez GY, Cervera Montejano M.D. Etnoteorías y prácticas de lactancia materna en una comunidad maya de Yucatán. Estudios de Antropología Biológica. 2013;16:907-928.

57. Olivé L. Multiculturalismo y pluralismo. México: Paidós; 1999.

58. Organización Panamericana de la Salud, Organización Mundial de la Salud. La alimentación del lactante y del niño pequeño: capítulo Modelo para libros de texto dirigidos a estudiantes de medicina y otras ciencias de la salud. Washington DC: Organización Panamericana de la Salud; 2010.

59. Aisenson Kogan A. Cuerpo y persona: Filosofía y psicología del cuerpo vivido. México: Fondo de Cultura Económica; 1981.

60. Massó Guijarro E. Lactancia materna y revolución, o la teta como insumisión biocultural: calostro, cuerpo y cuidado. Dilemata. 2013;5(11):169-206.

61. Pérez-Gil Romo SE., Rueda Arroniz, F, Ysunza Ogazón A, Andrade Contreras M. Principales aspectos socioculturales relacionados con la lactancia en Malinalco, edo. de México. Archivos Latinoamericanos de Nutrición. 1991;41(2):182-196.
62. Morin E. La vía: Para el futuro de la humanidad. Barcelona: Paidós; 2011.

63. Kuhn TS. La estructura de las revoluciones científicas. México: Fondo de Cultura Económica; 1996.

64. Morin E. Ciencia con consciencia. Barcelona: Anthropos Editorial del Hombre; 1984.

65. Morin E. Introducción al pensamiento complejo. Barcelona: Gedisa Editorial; 1996.

66. Vilar S. La nueva racionalidad: Comprender la complejidad con métodos transdisciplinarios. Barcelona: Editorial Kairós; 1997.

67. Pérez-Gil SE, Díez-Urdanivia S, Vega A. El proceso y las prácticas alimentarias en mujeres de dos comunidades rurales de México. 1er Congreso Latinoamericano de Antropología; 11-15 jul 2005, Facultad de Humanidades y Artes, Rosario, Argentina.

68. Almeida-Filho N. Complejidad y transdisciplinariedad en el campo de la salud colectiva: evaluación de conceptos y aplicaciones. Salud Colectiva. 2006;2(2):123-146.

\section{FORMA DE CITAR}

Ramos Rodríguez RM, Hernández González MG. Tensiones y contradicciones en la intervención gubernamental para la promoción de la lactancia materna. Salud Colectiva. 2017;13(4):677-692. doi: 10.18294/sc.2017.1357

Recibido: 21 de marzo de 2017 | Versión final: 8 de junio de 2017 | Aprobado: 3 de agosto de 2017 